\title{
Design and application of Ad Hoc network system based on WIFI
}

\author{
Yueming Ming ${ }^{1, a, *}$ and Ze Zhang ${ }^{2, b}$ \\ ${ }^{1}$ College of Electronic Information Engineering, Inner Mongolia University, Hohhot, China \\ ${ }^{2}$ College of Electronic Information Engineering, Inner Mongolia University, Hohhot, China \\ ae283454903@qq.com, b zhangzeimu@163.com
}

Keywords: Ad Hoc Network ; Java simulation; AODV ; Hardware Node

Abstract. This paper outlines the concept of Ad hoc networks and analysis of this type of network and application prospects of further development of today's society. The main starting point for the study and analyze routing protocols AODV routing protocol from its operational mechanism. With the Eclipse development platform and Jave programming language of their algorithm as the core routing protocol for simulation. The routing algorithm is applied to study, propose a hardware-based WIFI node and acquisition can be used as meteorological conditions, the test can last for networking requirements.

\section{Introduction}

The computer technology, of which the Internet is the representative has been showing wider and wider application and its skills are getting more and more completed in 21 centrary, expecially in this service industry whose character is speediness and convenience, the consumers prefer a life sytle, in which they can use WIFI to surf the Internet wherever and whenever. However, with the significant increasing demand for WIFI, it is that how to break the conventional service spot and supply larger space cover to satisfy people with rapid and convenient connection with the Internet has exposed higher requirement [1].Also, when we lack correspondence infrastructure and urgent corresponce is needed, how can we accomplish the information collection work on the mobile node. So, people start to explore how to build the temporary WIFI to solve the problem.

Transmittal mode of conventional WIFI is much limited. In this mode, it requires a central node, such as base station, in its cover space, for example celluler network, in which, the digitals are transmitted through base station via wires and then, to the target corresponding node. It needs many base stations in conventional WIFI, which causes engeneer costs and operation costs[2]. Then, there are no such drawbacks in the newly striving wireless multihop sensor network.Fig.1 show that MANET contrasts with traditional network In this new network, every node plays two important roles. First, every node acts as sending terminal and receiving terminal, second, it replaces base station in conventional WIFI and responsible for the disposal and forwarding function of information, ie, the function of Router[3].

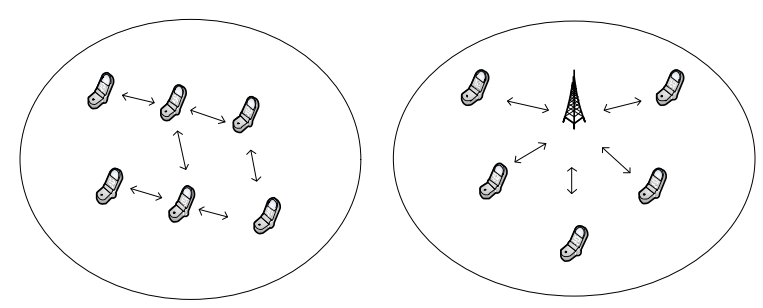

Figure.1 The MANET contrast with the traditional network 


\section{Brief Introduciton of Ad Hoc}

\section{Network characteristcs}

The outstanding characteristic of this network is its independent on infrastructures and it is comprised of mobile nodes. They are equal and independent to each other. The node, acting as mainframe, collects and sends information, in the meantime, finishes information transmittion, acting as Router[4]. The characteristics of Ad Hoc network as follows:

1. no center: equal mainframes, no central node[5].

2. dynamic topology: the nodes in the network is not stationary, once the position of node changes, the connection ways among them will change, ie, the topological structure is not stationary.

3. multi-hop routing: Thousands of mobile nodes exist in Ad Hoc network, when these nodes dot in a large restrict, the transmission is limited by transmittal distance, so, if we realize information transmission between two nodes, we have to use a terminal that can connect the two nodes and transmit information between the two nodes.

4. limited energy: as a network technology, it is inconvenient to supply electricity directly, and especially in remote areas, due to rare labor forces, it is extremely inconvenient to change electricity supplying facilities.

5. better flexibility: the network can be set anywhere, also, the addition and omission of node is at will, so, the network is more flexible.

6. strong survivability: there is no central controlling node in the network, so, a paralyzing node will not paralyze the whole network[6].

\section{Application background of the network}

Conventional network technology gets more developed, and how to solve these problems existing in the conventional network has become hot in technological exploration. Many advantages of Ad Hoc network are complementary to some drawbacks in conventional network, so, we can apply this technology to many fields:

1.Military communication: from conventional war to contemporary digital war, the distinguished difference lies in digital corresponding and remote control. If the command center is under attack, the whole troops will be paralyzed, but if the troops are facilited with Ad Hoc, even the base station is destroyed, the corresponding facilities can work without effect[7].

2. Emergence service: when we encounter natural disaster, fixed communication network cannot work normally, so we can supply a temporary communication to quickly recover the communicaiton, which bring basic service for dimentional salvage and is of great use value.

3. Mobile meeting: in temporary outdoor environment, we can establish a temporary network by using Ad Hoc network, to coorporate several distributed meetings.

4. Sensor network: when terminal node acts as sensor, it still realizes the real-time transportation of communication data collected by sensor, and supply service for fire control, guidance and ecological environment monitoring.

\section{AODV routing protocol}

Its significant characterization is that it carries out the routing seeking when requires, in the meantime, a reverse routing is erected. The reverse routing can to some extent retarts the effect of broadcast storm and improve the routing delay. AODV protocol not only realizes unicast, but also multicast. It changes the routing information at regular time in the DSDV, so when there is data at the node needed to transport, the routing request is sent to carry out the data transportation[8]. So, when there is no data to transport, the node is at rest. In the protocol, the required routes are activated and there is no need to manage the other nodes, that is why in this managing mechanism we decrease the broadcast ammount.

Fig.2 shows that the AODV running process. When the node in the network receives a routing request, a reverse access to originating node is established, so when the node sends data to originating node, there is no need for repeated check. It is the convenient and effective characterization that helps 
establish the function. After verifying the node, a further step will be taken. If this node the one the originating node needs, the origination node will receive the response message from the needed node, which notifies the originating node that an aiming route has been found, in this way, the originating node stops finding the aiming route it needs till a response message returns. If the above cannot be carried out, the requesting broadcast is activated to accomplish searching route.

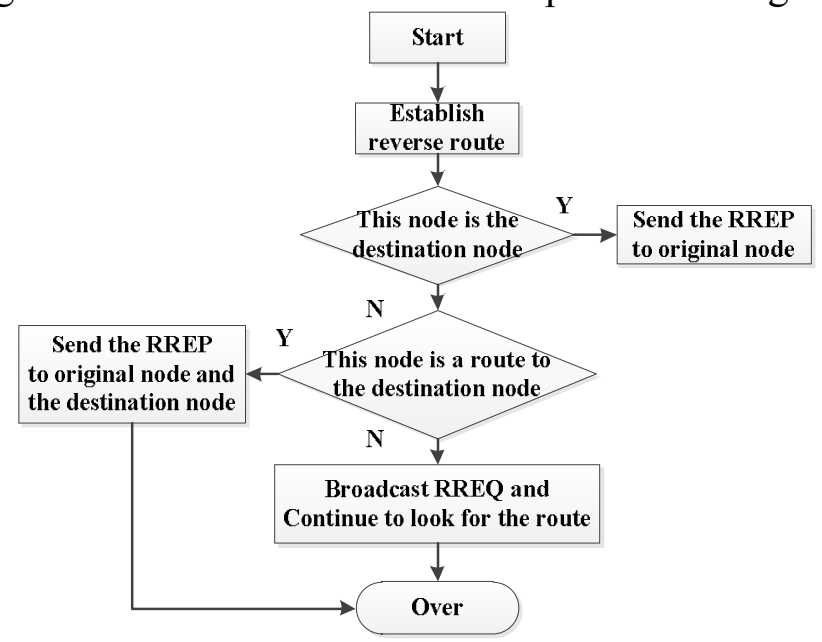

Figure. 2 AODV running process

The task of Network protocol is to complete information transportation by searching a suitable route. When the protocol starts to work, it receives information files.

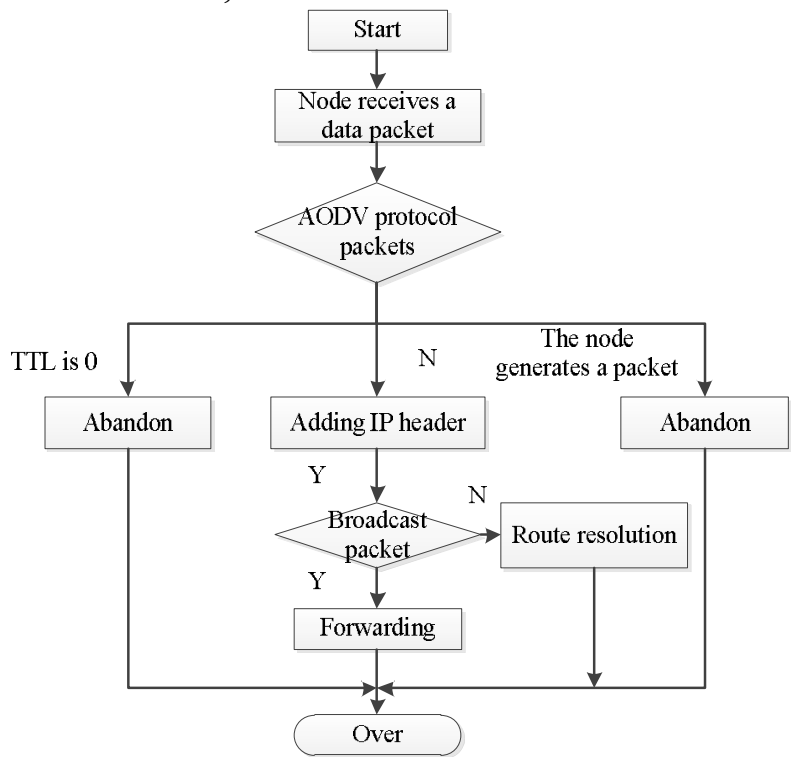

Figure.3 AODV rec-function running flowchart

The first step to tackle thses information files is to verify whether they belong to AODV protocol files. After verifying that they fit protocol rules, the disposal of the information file will be carried out. The treatment scheme of recv function in AODV is showed as the Fig.3[9].

\section{Algorithm and simulation realization}

\section{Maitenance of serial number}

If an aiming node is added itsefl a serial number, it can stop the collision between the request and the reverse routing sent by RREQ message. The route tackles the added serial number through an unsigned way. when the serial number cycles and reaches the biggest 32 bit integer variable 4294967295, the resulting number of continued addition is 0 . Whether the information at the node is new or old can be made sure through comparising the temporary serial number and the newly received serial number. In the comparison, the 32 bit integer variable is adopted, which is needed in the circle of serial number. 
When the subtract result of two serial numbers is under 0 , we can judge the temporary serial number is older than the new one, so the newly received routing information will be given up[10].

\section{Routing table}

When the information files are transported to other nodes through the intermediate node, the functioning routes will renew their validity, after renewing the validity, the time will also change, which happens because of ACTIVE_ROUTE_TIMEOUT, so the temporary time is calculated by adding the delayed time to the originating time. Based on the equal position principle of corresponding, the upstream node should change its validity by adding ACTIVE_ROUTE_TIMEOUT between the nodes. Once the route works, the validity will change[11] .

\section{Production of routing request}

RREQ-ID code and starting IP site will be cached in the start site before the route sends request. After a period of cache, the RREQ broadcast will be started, and the delayed time depends on the longation of PATH_DISCOVERY_TIME. If a node receives RREQ ID code and IP site which are the same with its, then the node will give up the request file, because the file could be the one sent by itself before.

\section{Controling transportation of request message}

First, a primary value TTL_START is set for TTL in the IP in the request file, and the longest responding time is set as NET_TRAVERSAL_TIME. If there is no response after sending a routing request, now the RREQ time is overdue, and the node will again send a RREQ message to search for the route. The accumulation process will be repeated till TTL reaches its peak TTL_THRESHOLD. When it reaches the peak, every TTL value will be set as the NET_DIAMETER.

\section{Production routing response}

When some node produces a routing response message, the node will copy the serial number of the originating node and IP site of the aiming node in the routing request message to the corresponding site of routing response. Once the RREQ is established, the routing response message is transported to down-stream node, which is usually the down-stream node leading to originating node. After the originating node receives the RREQ, every time the routing response message is transported, the value will be added 1. through such record method, we know the hop count between the originating node and the aiming node.

\section{Generating Route Reply}

After the node receives a routing response, it will, through the longest matched way, search a upstream route. When it is necessary to search route through this way, then we can establish an ineffective route through a upstream node. When other nodes send data through this node, the hop count of RREQ is added by one. Now we call it new hop count. If it can not search the upstream route before tha aiming node, a new route will be established.

\section{Hello message}

A node can get a linkage information through sending a Hello message. When there is an active node in the network, then it will broadcast a Hello. Every regular interval, a node will check whether there is a Hello message in a particular interval. If there is no Hello message, this node will broadcast a RREQ message, the TTL value in the message is one, which a actually the Hello message.

\section{RERR message, route overdue and route deletion}

The wrong messages in route are sent in two modes: broadcast and unicast. When the broadcast can not be carried out, the node will send the unicast to previous node. When RERR is sent to previous node by unicast, the message is regarded as a controlling message, and the RERR message generated in every second can not exceed RERR_RATELIMIT.

\section{The action after reset}

After a reset node becomes effective route which goes through a deletion period, the node will not be regarded the next hop by other nearby nodes. Once the node receives the RREQ message from other nodes, the serial number of the node will be added one, for which the reason is that the routing request message brings the biggest serial number of goal node to this node. If this node do not receive the 
RREQ message data file, then this node will initialize the inner serial book, the serial number becomes zero[12].

\section{Simulation realization and verification}

The software platform has the function in which it establishes Ad-Hoc network nodes, fullfils the communication connection and finishes the Ad-Hoc network dynamic visible process. So, we proclaim request and verification to that whether the algorithmics compiling are reliable and steady in the routing protocol operating mechnism. After establishing the software platform, in order to verify its function, we can simulate real scenes, and set nodes with different distances and quantities in the established node network district. We choose several nodes in the operating district, and continue debugging the already-realized-aiming functions in the compiling process, so in order to operate conveniently, we only set four-node network in the verification, and the inititive cover of the nodes is 150 meter.

\section{Application case}

Automatic weather station, as a application with mature technologies, abtains information mainly relying on precise instruments and remote wireless communicaiton facilities such as satellite, so automatic weather stations cannot be set densely, and the areas are mainly spacious. In terms of this condition, thd monitoring of sudden natural disasters in remote and special areas is not in time and effective. So, we can use Ad Hoc network nodes as sensors, and apply these sensors to monitor the environment, of which the advantage can make up for the disadvantages of automatic weather stations. We can use the network to monitor related weather information in local restrict as our main goal, and repidly tackle and transport weather data, realizing goal of intelligence, informatization and automation, in the meantime, we can collect and directly display information.

Fig.4 shows that the node hardware block diagram.In the chapter of Application case we accomplished the design of hardware node independenty. The designed hardware node can be used for weather monitoring, and the designed nodes can all, as an independent part, finish task of collection of related weather information, such as, weather, pressure, precipitation, humidity, and wind speed and direction.

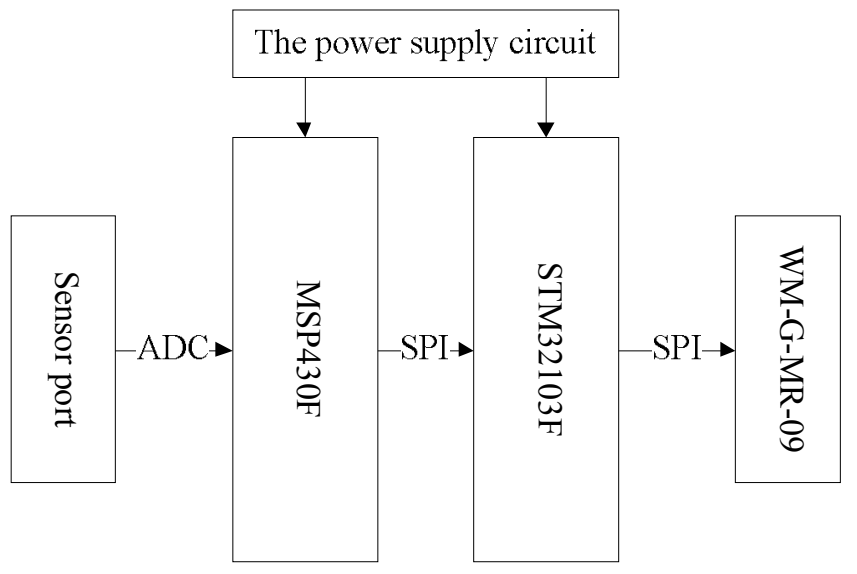

Figure.4 Node hardware block diagram

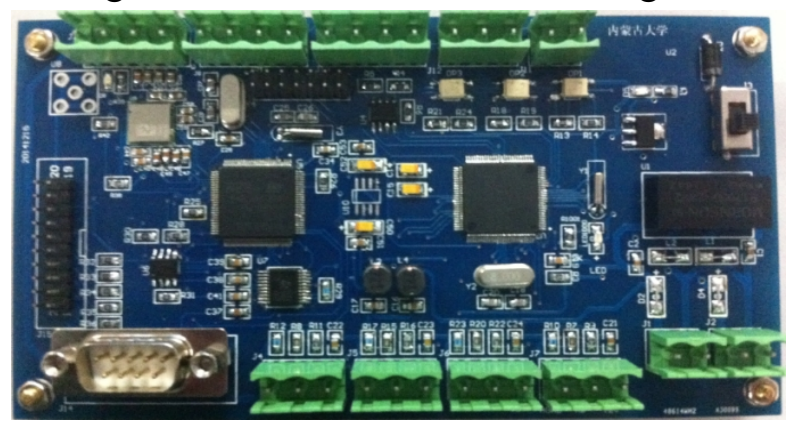

Figure.5. The realy node picture 
The application nodes usually distribute in remote areas, which puts high inquirement on power supply capacity of hardware, so, we choose ultra-low power consumption chip MSP430 as the chip to realize the data collection. In the meantime, we choose STM series chips to drive WIFI for data transportation and reception function in networking[13].Fig.5 shows that the realy node picture.

\section{Conclusions}

The article mainly states the features and application background of Ad Hoc network, and emphasizes the analisis of the three message modes of AODV routing protocol and the operating algorithm of routing operating methanism. Based on this, a dynamic simulation software that can operates routing algorithm is produced, and the display function of dynamic networking of Ad Hoc Network is realized, in the meantime, the operability of algorithm is verified.. At last, the application study is proposed, a group of hardware nodes collecting weather data are designed. The network layer operates the routing protocol and algorithm, realizes the related function of node network and transporting data, which again verifies the practice of algorithm, and in the meantime, accomplishs the function requirement of data collection in given environment.

\section{References}

[1] Xuefeng Dan, Jian Hu. Structure wireless ad-hoc network[J]. Ship Electronic Engineering, 2012, 32(4):83-84. "In Chinese"

[2] Huijie Zhang, Ding Zhong. Cortex-M3-based design of automatic weather stations[J]. Electronic Technology,2012, (08):70-72. "In Chinese"

[3] Boukerche, A. Wireless multimedia sensor and actuator system: A necessary public security and safety tested for an urban emergency preparedness class of applications[C], IEEE/ACS International Conference on Computer Systems and Applications (AICCSA), 2010, 16-19.

[4] Kefu Wang. Ad Hoc network Key Technology and Application.[J].Guang Dong Communication Technology, 2006, 25(11):34-37. "In Chinese"

[5] Xuefei Wang. Second-generation mobile ADHOC network routing analysis[J]. Journal of Huangshan University, 2005, (06):63-67. "In Chinese"

[6] Ling Xiao, Renfa Li,Juan Luo. Summary of simulation of VANET[J]. Journal of System Simulation, 2009, (17):5330-5335. "In Chinese"

[7] Gregory J Potti, William J Kaiser. Wireless integrated network sensors[J]. Communications of the ACM, 2000, 43(5): 51-58.

[8] Haitao Wang, Shaoren Zheng. Mobile Ad Hoc Network Routing Protocols and Performance Comparison[J].Chong Qing Jurnal of Posts and Telecommunications. "In Chinese"

[9] Written by Linxing Chen, Yi Cao, Xi Zeng. Mobile Ad Hoc Network[M]. Beijing Electronic Industry Press, 2006.4. "In Chinese"

[10] Chao Li.TCP/IP Architecture transport layer protocol analysis[J]. Information and Communication,2008. "In Chinese"

[11] Written by Limin Sun, Jianzhong Li. Wireless sensor networks[M].BeiJing, Tsinghua University Press.2005.4.10“In Chinese"

[12] Chasong Deng, Changping Zhu, Qingbang Han. Mine safety monitoring terminal based on embedded Wi-Fi technology [M]. Modern electronic technology 2010. "In Chinese" 
[13] Achtzehn A, Meshkova Ansari J, Mahonen P. A Scalable Sensor Network Testbed for Rapid Protocol Performance Evaluation[C]. IEEE Communications Society Conference on Sensor, Mesh and Ad Hoc Communications and Networks Workshops, 2009, 1-3. 